Original Research

\title{
Theoretical investigation of the single and double ionization spectra of $\mathrm{M}(\mathrm{CO})_{6}, \mathrm{M}=\mathrm{W}$ and $\mathrm{Cr}$
}

Behnam Nikoobakht ${ }^{1,}{ }^{*}$, Gulzari L. Malli ${ }^{2}$, Martin Siegert ${ }^{3}$

1. School of Chemistry, The University of Sydney, Sydney, Australia; E-Mail: bnikoobakht@gmail.com.

2. Department of Chemistry, Simon Fraser University, Burnaby, British Columbia V5A 1S6, Canada; E-Mail: malli@sfu.ca.

3. IT services,Simon Fraser University, Burnaby, BC, Canada V5A 1S6, Canada; E-Mail: siegert@sfu.ca.

* Correspondence: Behnam Nikoobakht; E-Mail: bnikoobakht@gmail.com

Academic Editor: Maxim L. Kuznetsov

Special Issue: Coordination Chemistry and Metal Complexes

Advances in Chemical Research

2020, volume 2, issue 4

doi:10.21926/acr.2004010
Received: April 26, 2020

Accepted: November 10, 2020

Published: December 14, 2020

\begin{abstract}
In this work, we study the single and double ionization spectra of the $\mathrm{M}(\mathrm{CO})_{6}$, with $\mathrm{M}=$ (W and $\mathrm{Cr}$ ) complexes by applying the four-component algebraic diagrammatic construction and Fock-space coupled cluster methods to extend earlier studies based on less demanding approaches. The computed single and double ionization potentials are in good agreement comparing with the available experimental results. The electronic structures of the cationic molecular systems are carefully investigated by computing accurately single and double ionization potentials. The final state characterization is relied on group theoretical considerations of the contributing orbitals and allowed for a clear assignment. Energy level diagrams show the effect of spin-orbit (SO) coupling starting from scalar relativistic results and for the heavy representative $\mathrm{M}(\mathrm{CO})_{6}$ with $\mathrm{M}=(\mathrm{W}$ and $\mathrm{Cr})$ nonadditivity effects of the SO and electron correlation can be observed requiring a consistent treatment of both contributions.
\end{abstract}

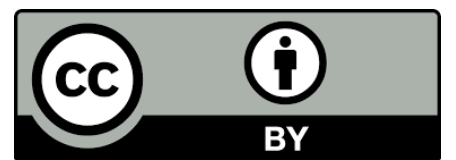

(C) 2020 by the author. This is an open access article distributed under the conditions of the Creative Commons by Attribution License, which permits unrestricted use, distribution, and reproduction in any medium or format, provided the original work is correctly cited. 


\section{Keywords}

Spectroscopy; gas phase; single ionization potential; double ionization potential; electronic structure calculation; four-component electronic structure calculation

\section{Introduction}

Photoelectron spectra provide an accurate way for a thorough exploration of the electronic structure of molecular systems. The electronic structure of transition-metal complexes is of particular interest due to the complexity of the bonding and the nature of the orbitals involved [1]. Photoelectron spectra of transition metal complexes can be studied by $(a)$ energy and intensity analysis and molecular orbital assignments of the valence levels, which can often be employed to gain detailed information of the bonding; $(b)$ energy and intensity analysis of core electron excitation bands, which have bearings on the ionization dynamics in terms of relaxation and charge transfer screening; and (c) vibronic analysis of individual electronic bands yielding knowledge relating to bonding and dynamics [2]. Theoretically, studying the electronic structure of the highly symmetric transition metal complexes is interesting, due to presences of the Jahn-Teller (JT) effect and spin-orbit (SO) coupling. These two important effects play important roles in studying the electronic structure of metal complexes $\mathrm{M}(\mathrm{CO})_{6}$ with $\mathrm{M}=(\mathrm{W}$ and $\mathrm{Cr}$ ) complexes, which constitute the main topics of the current work.

As indicated, we consider the transition metal complexes, in which $\mathrm{CO}$ molecules adsorbed on metal surfaces of $\mathrm{W}$ and $\mathrm{Cr}$. Experimental photoelectron spectra for these two complexes were reported in the literature $[3,4]$. For $\mathrm{W}(\mathrm{CO})_{6}$, the photoelectron spectrum was measured in different energy ranges. There are some differences between the two reported experimental photoelectron spectra in the literature. Higginson et al measured spectrum in energy range of 7 to 20 [3] and in a recent study by Lei et al, they measured the photoelectron spectrum in the energy range of 5 to $25 \mathrm{eV}$ [4]. The intensities and broadening of bands of the two measured photoelectron spectra of $\mathrm{W}(\mathrm{CO})_{6}$ reported in Refs. [3, 4] are different. Fukuda et al computed the photoelectron spectrum of $\mathrm{W}(\mathrm{CO})_{6}$. Their results were in a good agreement with the experimental result presented in Ref. [3] but were partially in agreement with the experimental result in Ref. [4], because the computed photoelectron spectrum by Fukuda et al did not cover the whole energy range of the recent experimental photoelectron spectrum reported in Ref. [4]. Furthermore, no theoretical investigations relating to the evaluations of the double ionization potentials (DIPs) have been reported in the literature for $\mathrm{M}(\mathrm{CO})_{6}$ with $\mathrm{M}=(\mathrm{W}$ and $\mathrm{Cr}$ ) yet. In this work, our motivation is to readdress the photoelectron spectra of the $\mathrm{M}(\mathrm{CO})_{6}, \mathrm{M}=$ (W and $\mathrm{Cr}$ ) complexes by applying four-component algebraic diagrammatic construction (ADC) and Fock-space coupled cluster (FSCC) methods in order to obtain more accurate information on the electronic structure of these complexes to explain the partial difference between the computed and measured photoelectron spectra. Also, the earlier studies would be extended by computing the double ionization spectra of these molecular systems using the four-component $\operatorname{ADC}(2)$ method.

The lowest electronic state of ${ }^{2} \mathrm{~T}_{2 \mathrm{~g}}$ of $\mathrm{M}(\mathrm{CO})_{6}$ with $\mathrm{M}=(\mathrm{W}$ and $\mathrm{Cr})$ complexes in the degenerated shell has configuration $t_{2 g}^{5}$. It is well-known that the JT effect can lift the degeneracy 
of the degenerate ground state when the distortion along the JT active modes takes place [5]. Due to degeneracy in the ${ }^{2} \mathrm{~T}_{2 \mathrm{~g}}$ electronic ground state, one would expect that this molecule shows the $\mathrm{JT}$ activity resulting in lifting the degeneracy in the ${ }^{2} \mathrm{~T}_{2 \mathrm{~g}}$ electronic ground state. The JT active modes are $e_{g}$ and $t_{2 g}$. Hence, this leads to the well-known JT problem of type $T_{2 g} \otimes\left(e_{g} \bigoplus t_{2 g}\right)$ in these complexes [5]. This indicates that the five-fold degenerate d-orbital on the transition metal is separated into a three fold degenerate orbital $\mathrm{T}_{2 \mathrm{~g}}$ symmetry and a two fold degenerate orbital of $E_{\mathrm{g}}$. Considering the electron spin leads to the ten double valued-reducible representation $\left(T_{2 g}+E_{g}\right) \times E_{g 1 / 2}$, which can be decomposed into irreducible representations of double point group $\mathrm{E}_{\mathrm{g} 5 / 2}+2 \mathrm{G}_{\mathrm{g} 3 / 2}$. The $\mathrm{E}_{\mathrm{g} 1 / 2}$ and $\mathrm{E}_{\mathrm{g} 5 / 2}$ are two-dimensional double-valued irreducible representations. The $G_{\mathrm{g} 3 / 2}$ is a four-dimensional double-valued irreducible representation. In the decomposed irreducible representations $E_{\mathrm{g} 5 / 2}+2 \mathrm{G}_{\mathrm{g} 3 / 2}$, one of the two $\mathrm{G}_{\mathrm{g} 3 / 2}$ manifolds corresponds to the ${ }^{2} \mathrm{E}_{\mathrm{g}}$ state while the other $\mathrm{G}_{\mathrm{g} 3 / 2}$ and $\mathrm{E}_{\mathrm{g} 5 / 2}$ are irreducible representations of the ${ }^{2} \mathrm{~T}_{2 \mathrm{~g}}$ state. Thus, inclusion of electron spin lifts partially the six-fold degenerate electronic state ${ }^{2} \mathrm{~T}_{2 \mathrm{~g}}$ into four-fold and two-fold degenerate electronic states in these open-shell systems. In this work, we mainly consider the spin-orbit (SO) coupling for analyzing and computation of the single and double ionization spectra of these complexes and we believe that the JT effect has a minor role for investigation of the electronic structure of these complexes. The reason is that the SO coupling is relatively strong and it nearly vanishes the contribution of the JT effect due to a quenching between the SO and JT couplings (the Ham effect) $[5,6]$.

\section{Computational Details}

$\mathrm{M}(\mathrm{CO})_{6}$ with $\mathrm{M}=(\mathrm{W}$ and $\mathrm{Cr})$ complexes are optimized in the $\mathrm{O}_{\mathrm{h}}$ symmetry point group by using a dyall.v3z basis set for all atoms with the large and small basis sets 24s19p12d9f and $19 s 36 p 28 d 12 f 9 g$, respectively. The total number of the primitive gaussians is 1983 . The lengths large and small components are 583 and 1400. respectively [7]. Optimizations are carried out by employing the four-component density functional theory (DFT)/B3LYP method as implemented in the DIRAC14 package [8]. Here, B3LYP stands for Becke's three-parameter DFT hybrid exchange functional (B3) with the Lee, Yang, and Parr41 (LYP) dynamical correlation functional. Within the ADF program package [9], we also carry out the optimization using the zeroth-order regular approximation, ZORA, 2c Hamiltonian treating the SO effect (SO ZORA) [10-12]. In this calculation, the B3LYP functional and the TZ2P basis set [13] are employed. For completeness, we perform the ADF calculations using scalar-relativistic (SR ZORA) and nonrelativistic (NR) Hamiltonians with all electrons included. In Table 1, we report the calculated bond lengths at the different levels of theory and compare them with experimental values.

Table 1 The calculated equilibrium values of the bond lengths at different levels of theory, which is compared by the previous reported values in the literature shown in parentheses.

\begin{tabular}{|c|c|c|c|c|c|c|}
\hline & distance & SO-ZORA & SR-ZORA & NR & expt. & 4c-DFT \\
\hline $\mathrm{W}(\mathrm{CO})_{6}$ & $W-C$ & $\begin{array}{l}2.062^{\mathrm{a}} \\
\left(2.062^{\mathrm{b}}\right)\end{array}$ & $2.29^{a}$ & $2.094^{a}$ & $2.058^{c}$ & $\begin{array}{l}2.057^{\mathrm{a}} \\
\left(2.062^{\mathrm{d}}\right)\end{array}$ \\
\hline
\end{tabular}




\begin{tabular}{ccccccc} 
& $\mathrm{C}-\mathrm{O}$ & $\begin{array}{l}1.154^{\mathrm{a}} \\
\left(1.154^{\mathrm{b}}\right)\end{array}$ & $1.154^{\mathrm{a}}$ & $1.152^{\mathrm{a}}$ & $1.148^{\mathrm{c}}$ & $\begin{array}{l}1.153^{\mathrm{a}} \\
\left(1.153^{\mathrm{d}}\right)\end{array}$ \\
\hline $\mathrm{Cr}(\mathrm{CO})_{6}$ & $\mathrm{Cr}-\mathrm{C}$ & $1.912^{\mathrm{a}}$ & $1.909^{\mathrm{a}}$ & $1.909^{\mathrm{a}}$ & $1.918^{\mathrm{b}}$ & $1.906^{\mathrm{a}}$ \\
& $\mathrm{C}-\mathrm{O}$ & $1.153^{\mathrm{a}}$ & $1.153^{\mathrm{a}}$ & $1.153^{\mathrm{a}}$ & $1.141^{\mathrm{b}}$ & $1.157^{\mathrm{a}}$ \\
\hline
\end{tabular}

${ }^{\text {aT }}$ The computed values are from this work.

${ }^{\text {b}}$ The values are taken from Ref. [14]

'The experimental values are taken from Ref. [15]

${ }^{\mathrm{d}}$ The values are taken from Ref. [16]

For evaluating the single and double ionization spectra, the electron correlation and SO coupling effects are considered consistently, which are achieved by the DC-FSCC [17] and DC$A D C(3)[18]$ approaches using the Dirac-Coulomb (DC) Hamiltonian,

$$
H_{D C}=\sum_{i=1}^{N}\left(c \alpha_{i} \cdot p_{i}+\beta_{i} m_{e} c^{2}+V(i) I_{4}\right)+\sum_{i<j}^{N} \frac{1}{r_{i j}}
$$

where the potential $V(i)$ due to $\mathrm{n}$ finite nuclei of the molecular system is equal to the sum of their nuclear potentials, i.e. $V(i)=\sum_{n} V_{n}$, and for molecular systems including heavy atoms (with $Z>$ 70 ), the finite nuclear model is always employed [19]. We shall utilize the Gaussian nuclear model [8], in which a single Gaussian function is employed for each nuclear charge distribution. Contribution of SO coupling can be ignored by utilizing the scalar relativistic Hamiltonian based on a spin-free Hamiltonian calculation according to Dyall's formalism [20]. Comparing the results deduced from the spin-free Hamiltonian with the corresponding ones using the DC Hamiltonian reveals the importance of the SO coupling which provides a thorough information for better understanding of SIP and DIP spectra for these two complexes. The approach relied on the spinfree Hamiltonian takes into account all scalar relativistic effects and can therefore be seen as a Douglas-Kroll (DK) approach of infinite order. For each Hamiltonian the electronic structure method can be Hartree-Fock, coupled cluster and nonadditivity effects can also be revealed, if necessary. For our analysis, we performed DC-ADC(3) and DC-FSCC calculations and compared with the corresponding DK-ADC(3) and DK-FSCC results. In the case of DK-SCF and DC-SCF, koopmans' theorem was applied to neutral systems and the negative molecular orbital energies were specified with IPs. The DK-FSCC and DC-FSCC values correspond to the correlated cationic final state energies of the respective symmetry. As a result, the symmetry of a correlated final state can be specified by the zeroth-order term of the corresponding determinantal expansion having a single hole. The higher excited states possess the same symmetry.

All these methods are available in the DIRAC14 package [8] used for computing of the SIP and DIP spectra. In the relativistic framework, fully uncontracted basis sets have to be applied in order to generate the kinetically balanced small component basis. For all elements $\mathrm{W}, \mathrm{Cr}, \mathrm{C}$ and $\mathrm{O}$, the basis set dyall.v2z was employed in the evaluation of single and double ionization spectra [7, 21]. Due to considerable numerical effort we could not afford larger basis sets on the atomic centers. For the DC-FSCC method, the active spaces for $\mathrm{W}(\mathrm{CO})_{6}$ and $\operatorname{Cr}(\mathrm{CO})_{6}(-1,16 \mathrm{au})$ and $(-1,25 \mathrm{au})$, respectively. These indicate that the number of occupied and virtual spinors are 108 and 470 for 
$\mathrm{W}(\mathrm{CO})_{6}$, respectively and 108 and 574 , for $\mathrm{Cr}(\mathrm{CO})_{6}$, respectively. For the $\mathrm{DC}-\mathrm{ADC}(3)$ method, the active spaces for $\mathrm{W}(\mathrm{CO})_{6}$ and $\mathrm{Cr}(\mathrm{CO})_{6}$ are $(-1,1.6 \mathrm{au})$ and $(-1,1.16 \mathrm{au})$, respectively. These indicate that the number of occupied and virtual spinors are 108 and 190, respectively, for $\mathrm{W}(\mathrm{CO})_{6}$ and 108 and 164, respectively, for $\mathrm{Cr}(\mathrm{CO})_{6}$.

\section{Results and Discussion}

\section{$3.1 W(\mathrm{CO})_{6}$}

The ground state equilibrium geometry of $\mathrm{W}(\mathrm{CO})_{6}$ is computed at the NR, SR and SO-ZORA as well as the 4c-DFT levels of theory and compared with the previous computational and experimental results, which are summarized in Table 1 . The 4c-DFT results for both transition metal complexes are in good agreement with the corresponding experimental results.

The vibrational frequency calculation has been carried out using the ADF SO-ZORA and NR Hamiltonians for this complex ensuring that the optimized structure is in the minimum energy. It can be inferred from the assignment of the vibrational frequencies that they are either triply or doubly degenerate (with symmetry $T_{i j}$ with $i=1,2$ and $j=u, g$ as well as $E_{g}$ ) indicating of presence of the Jahn-Teller (JT) activity in the ground state of this highly symmetric molecule. The nomenclatures $g$ and $u$ refer to gerade and ungerade molecular orbitals. The two remaining modes are totally symmetric $A_{1 g}$. The computed vibrational frequencies are listed in Table 2 for this complex, where they are compared with the corresponding available experimental values. In general, the values obtained at the SO-ZORA level of theory are in a good agreement with the corresponding experimental and theoretical values reported in Ref. [14]. For $\mathrm{W}(\mathrm{CO})_{6}$, there are two experimental works in which the photoelectron spectrum of this molecule was measured in energy ranges ( 5 to $20 \mathrm{eV}$ ) and ( 5 to $25 \mathrm{eV}$ ) [4, 23]. The reported spectrum in Ref. [23] shows three bands, while the measured spectrum in Ref. [4] represents five bands. Furthermore, for these two measured spectra, there are differences in the intensity and broadening of the spectral bands especially for the band in the energy range of 12 to $17 \mathrm{eV}$. In principal, the main differences between these two measured spectra are in the middle and high energy parts of the spectra. Fukuda et al computed the photoelectron spectrum of this molecule using the $\mathrm{SAC}-\mathrm{Cl}$ (the symmetry-adapted cluster configuration interaction) method within the energy range of 5 to 20 eV [24]. Their results are in a good agreement with the experimental work of Ref. [23]. However, their work could not explain the inconsistency between the two experimental works reported in Refs. [4, 23]. In fact, the computational result reported by Fukuda et al was partially in agreement with the recent experimental result in Ref. [4] and the major differences are in the middle and high energy parts of the photoelectron spectrum. To remove this inconsistency and provide a clear understanding on the electronic structure of this complex, we carried out the SCF, ADC(3) and FSCC calculations using the DC and DK Hamiltonians as indicated in Sec. 2. The summaries of our results are shown in Figure 1, Figure 2 and Figure 3 as well as Table 3, which are described in more details in the following. In Figure 1, we show the energy level of $\mathrm{W}(\mathrm{CO})_{6}$ final states are computed using the $A D C(3)$ method employing the DC and DK Hamiltonians. The SO effect partially lifts the six-fold degenerate electronic state ${ }^{2} \mathrm{~T}_{2 \mathrm{~g}}$ into four-fold and two-fold degenerate electronic states in this open-shell system (see Figure 1). 


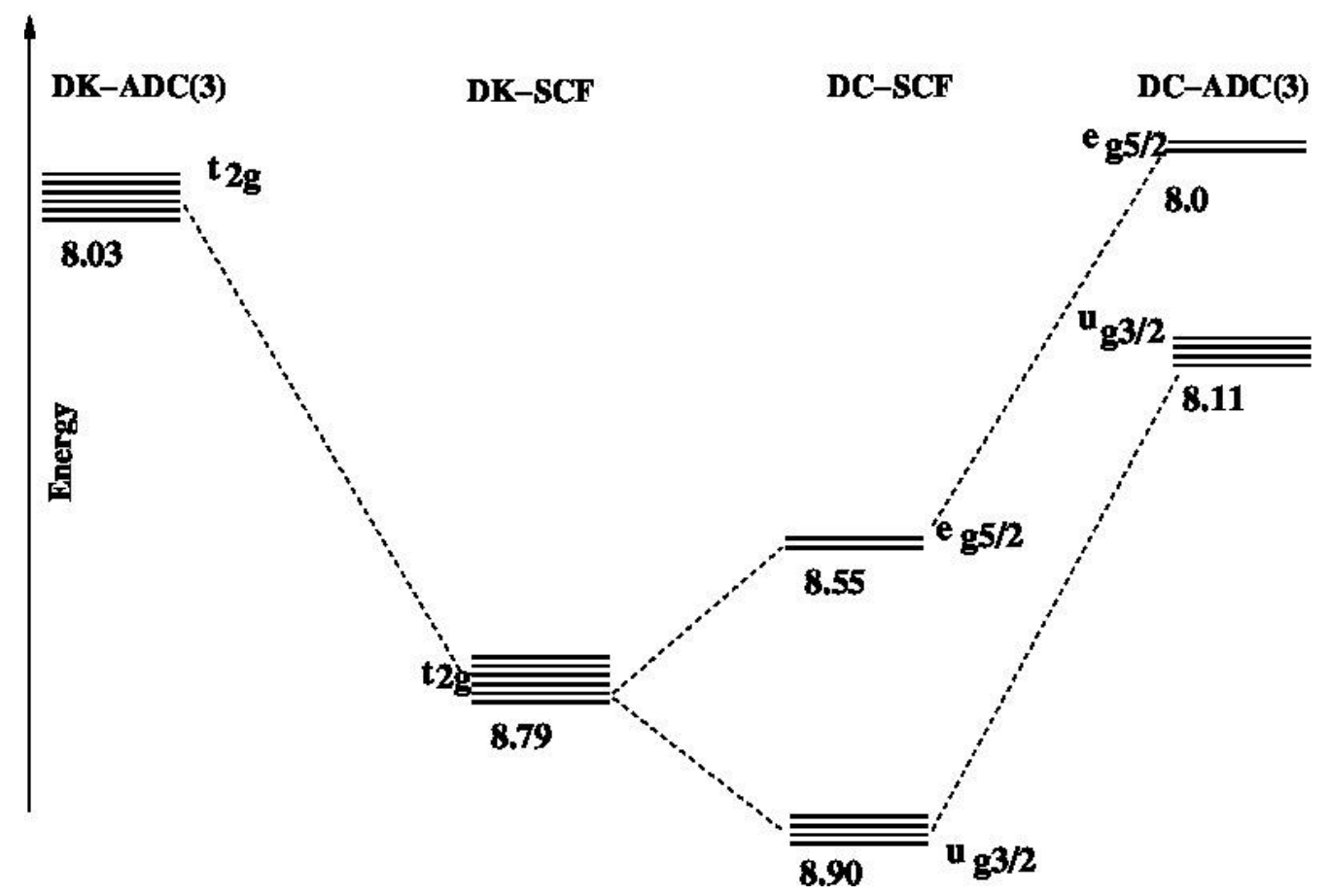

Figure 1 Energy level diagram for $\mathrm{W}(\mathrm{CO})_{6}$ calculated at the uncorrelated (SCF) and correlated $(A D C(3))$ level of theory for the DK and DC Hamiltonians in eV. The energy values correspond to the IPs.

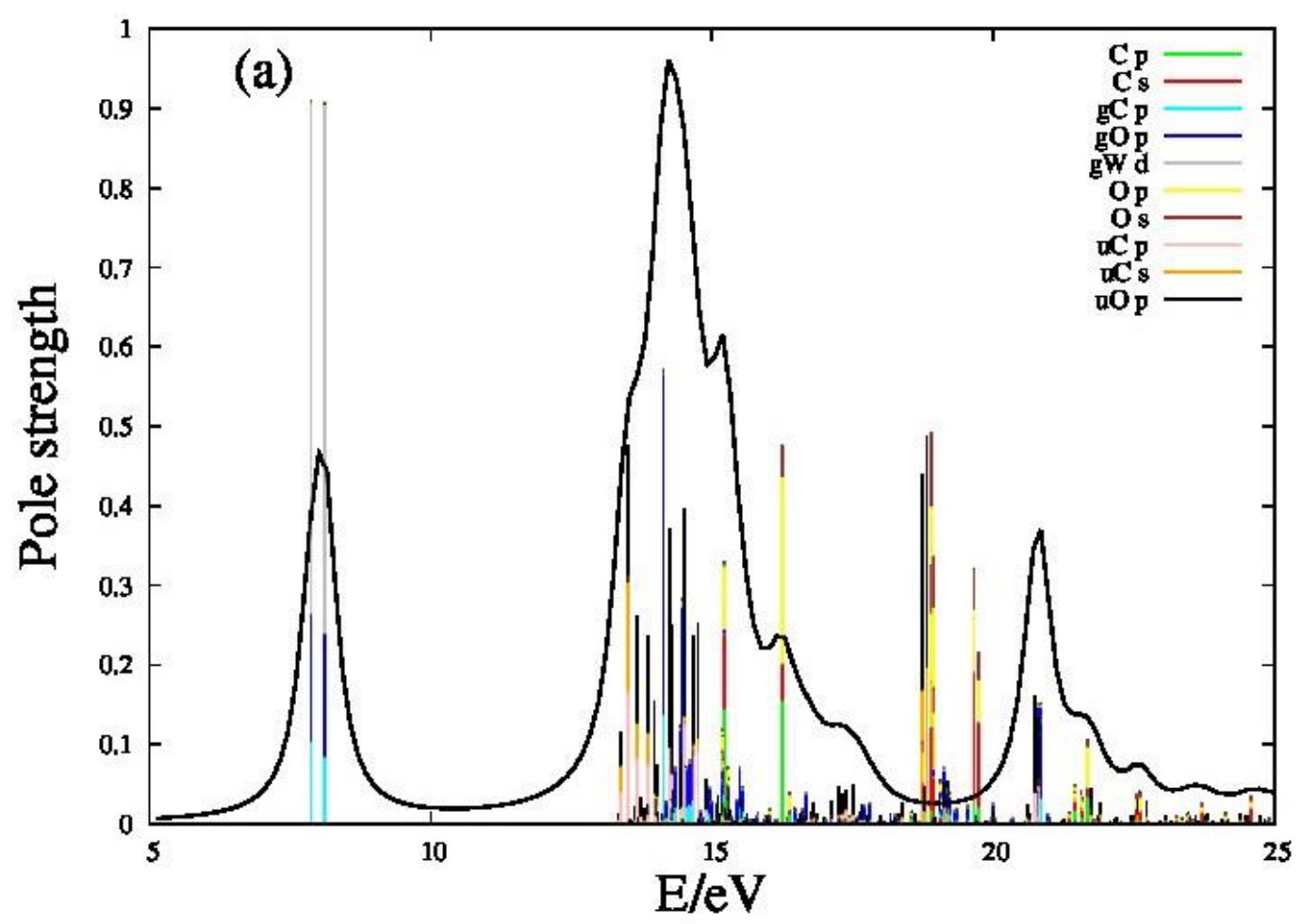

Figure 2 The simulated photoelectron spectrum of $\mathrm{W}(\mathrm{CO})_{6}$ calculated by employing the DC-ADC(3) method. 


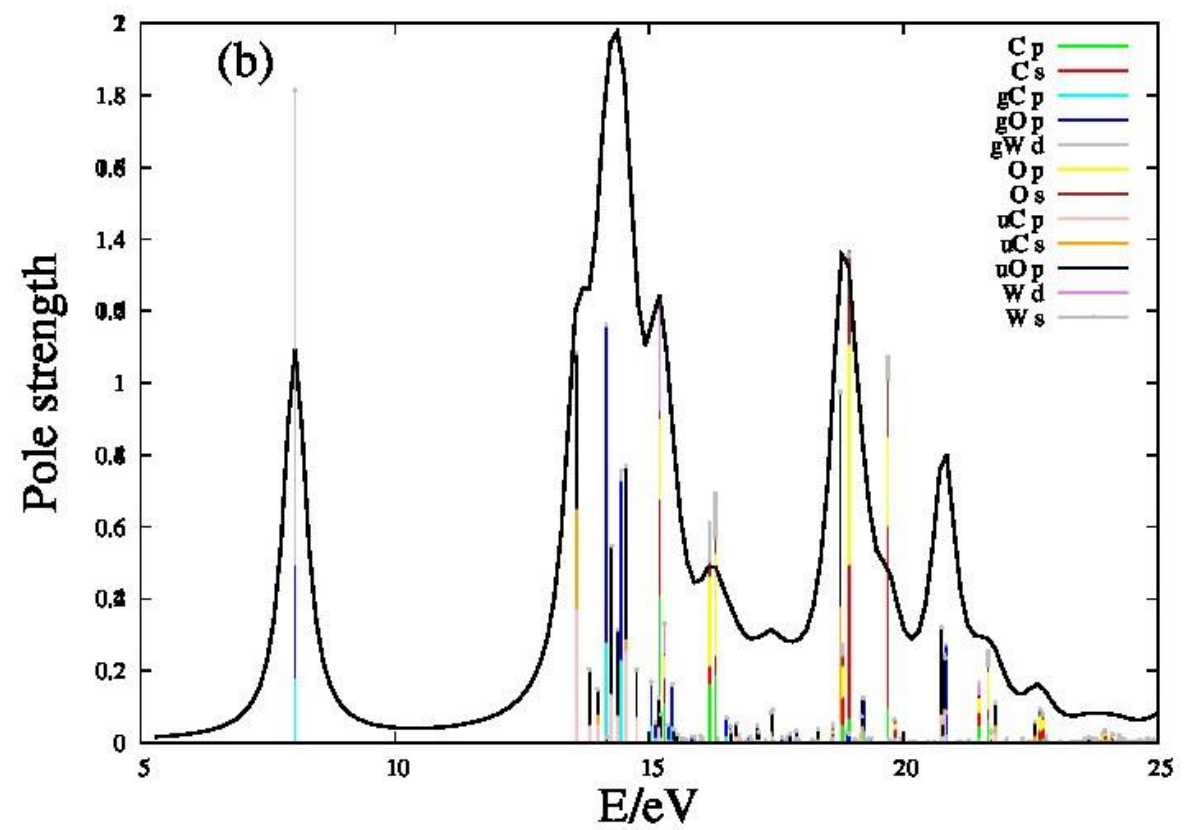

Figure 3 The simulated photoelectron spectrum of $\mathrm{W}(\mathrm{CO})_{6}$ calculated by employing the DK-ADC(3) method.

Table 2 Vibrational Frequencies for $\mathrm{W}(\mathrm{CO})_{6}$ computed using the ADF for the NR and SO-ZORA Hamiltonians, which are compared with the corresponding experimental values. The unit is $\mathrm{cm}^{-1}$.

\begin{tabular}{llcl}
\hline NR (this work) & SO-ZORA (this work) & Assignment & Expt. $^{\mathrm{a}}$ \\
\hline 72.8 & 38.6 & $\mathrm{~T}_{2 \mathrm{u}}$ & - \\
92.8 & 71.8 & $\mathrm{~T}_{2 \mathrm{~g}}$ & - \\
83.41 & 76.3 & $\mathrm{~T}_{1 \mathrm{u}}$ & - \\
375.25 & 363.3 & $\mathrm{~T}_{1 \mathrm{~g}}$ & - \\
372.33 & 379 & $\mathrm{~T}_{1 \mathrm{u}}$ & - \\
412.53 & 413.3 & $\mathrm{E}_{\mathrm{g}}$ & - \\
422.16 & 428.1 & $\mathrm{~A}_{1 \mathrm{~g}}$ & - \\
524.19 & 480.2 & $\mathrm{~T}_{2 \mathrm{~g}}$ & 482.2 \\
557.29 & 528.1 & $\mathrm{~T}_{2 \mathrm{u}}$ & - \\
609.22 & 591.5 & $\mathrm{~T}_{1 \mathrm{u}}$ & - \\
1980.67 & 1965 & $\mathrm{~T}_{1 \mathrm{u}}$ & 1997.6 \\
2003.71 & 1987.8 & $\mathrm{E}_{\mathrm{g}}$ & 2021.1 \\
2096.4 & 2085.8 & $\mathrm{~A}_{1 \mathrm{~g}}$ & 2126.2 \\
\hline
\end{tabular}

${ }^{\text {aRef.[22] }}$ 
Table 3 Ionization potentials of $\mathrm{W}(\mathrm{CO})_{6}$ for the valence spinors at the scalar and fully relativistic levels of theory which correspond to the DK and DC Hamiltonians, respectively. Note that these results are calculated by using $A D C(3)$ and FSCC methods. All values are in $\mathrm{eV}$. The values in the parentheses refer to the differences between the computed IPs with the corresponding experimental values reported in Ref. [23].

\begin{tabular}{|c|c|c|c|c|c|c|c|c|}
\hline state & $A D C(3)-D K$ & FSCC-DK & $A D C(3)-D C$ & FSCC-DC & $\mathrm{SAC}-\mathrm{Cl}^{\mathrm{a}}$ & OVGF $^{b}$ & Expt. $^{c}$ & Expt. $^{d}$ \\
\hline \multirow{2}{*}{$1^{2} \mathrm{~T}_{2 \mathrm{~g}}$} & 8.03 & 8.28 & $8.00(0.30)$ & 8.15 & $8.14(0.16)$ & 8.1 & 8.3 & \\
\hline & & & $8.11(0.45)$ & 8.35 & $8.37(0.19)$ & & 8.56 & 8.6 \\
\hline \multirow{2}{*}{$1^{2} \mathrm{~T}_{1 \mathrm{u}}$} & 13.57 & 13.91 & $13.32(-0.05)$ & 13.77 & $13.28(0.01)$ & 14.03 & 13.27 & 13.6 \\
\hline & & & 13.36 & 14.16 & 14.11 & & & \\
\hline \multirow{2}{*}{$1^{2} \mathrm{~T}_{1 \mathrm{~g}}$} & 13.82 & - & $13.91(0.29)$ & - & $14.38(0.18)$ & 15.42 & 14.2 & \\
\hline & & & 13.94 & - & 14.39 & & & \\
\hline \multirow{2}{*}{$1^{2} \mathrm{~T}_{2 \mathrm{u}}$} & 13.98 & - & $14.31(0.11)$ & - & $14.53(-0.11)$ & 15.55 & 14.42 & 14.3 \\
\hline & & & 14.31 & - & 14.53 & & & \\
\hline \multirow{2}{*}{$1^{2} \mathrm{~T}_{1 \mathrm{u}}$} & 14.15 & - & $14.56(0.32)$ & - & $14.76(0.12)$ & 15.8 & 14.88 & \\
\hline & & & 14.77 & - & 15.21 & & & \\
\hline \multirow{2}{*}{$2^{2} \mathrm{~T}_{2 \mathrm{~g}}$} & 14.17 & - & $14.95(0.25)$ & - & $14.97(0.23)$ & 16.04 & 15.2 & 15 \\
\hline & & & 14.98 & - & 14.99 & & & \\
\hline
\end{tabular}

${ }^{a}$ Ref. [24]

${ }^{\mathrm{b}}$ Ref. [25]

${ }^{\mathrm{C}}$ Ref. [23]

${ }^{\text {dRef. [4] }}$

In a DC-SCF calculation, due to the presence of the SO coupling, the $t_{2 g}$ orbital, composed of the $\mathrm{gW}_{\mathrm{d}}, \mathrm{gC}_{\mathrm{p}}$ and $\mathrm{gO}_{\mathrm{p}}$ orbitals, undergo a $\Delta_{1}=0.35 \mathrm{eV}$ splitting into the $\mathrm{E}_{\mathrm{g} 5 / 2}$ and $\mathrm{G}_{\mathrm{g} 3 / 2}$ spinors where the first mentioned is the highest occupied molecular orbital (HOMO). The reason for this considerable splitting can be explained by looking at the corresponding populations, where a significant $\mathrm{gW}_{\mathrm{d}}$ contribution of $70 \%$ in the DK cases is responsible for the splitting of the $\mathrm{t}_{2 \mathrm{~g}}$ orbitals compared to the $\mathrm{gW}_{\mathrm{d}}$ contribution of $67 \%$ and $70 \%$ in the $\mathrm{E}_{\mathrm{g} 5 / 2}$ and $\mathrm{G}_{\mathrm{g} 3 / 2}$ orbitals, respectively. This substantial $\mathrm{gW}_{\mathrm{d}}$ population of the ground state with $\mathrm{t}_{2 \mathrm{~g}}$ symmetry leads to the strong SO splitting of 0.24 and $0.11 \mathrm{eV}$ with respect to the DK value. After performing the SCF calculation at the scalar and four-component levels of theory, the corresponding ionised system was studied by the $A D C(3)$ and FSCC methods taking electron correlation into account. Our symmetry assignments based on the $A D C(3)$ method are also shown in Figure 1 . The first IP evaluated using the DK Hamiltonian is attributed to an ionization from the triply degenerate orbitals with $\mathrm{t}_{2 \mathrm{~g}}$ symmetry. When the SO coupling is taken into account by employing the DC Hamiltonian, these orbitals undergo a splitting and lead to two different IPs. By careful inspection of the underlying Mulliken population data and comparison with the corresponding 
transformation behavior in $\mathrm{O}_{\mathrm{h}}$ we can assign the first IP to $\mathrm{e}_{\mathrm{g} 5 / 2}$ and the second IP to $\mathrm{u}_{\mathrm{g} 3 / 2}$ It can be seen that the SO effect is significant for $1^{2} \mathrm{~T}_{2 \mathrm{~g}}$ in comparison with the $1^{2} \mathrm{~T}_{1 \mathrm{u}}$ state (see also Table 3). This observation is consistent with the population analysis of $1^{2} \mathrm{~T}_{2 \mathrm{~g}}$ where it shows that this state has the character of the $\mathrm{gW}_{\mathrm{d}}$, while $1^{2} \mathrm{~T}_{1 \mathrm{u}}$ has dominant contributions of the $\mathrm{uO}_{\mathrm{p}}, \mathrm{uC}_{\mathrm{s}}$, $\mathrm{uC}_{\mathrm{p}}$ characters (see Figure 2 and Figure 3). Thus the larger so splitting can be observed for the $1^{2} \mathrm{~T}_{2 \mathrm{~g}}$ state. It is instructive to compare the size of SO splitting computed using the $A D C(3)$ and FSCC methods using Figure 1 and Table 3. We mentioned that the $A D C(3)$ predicates the larger SO splitting for $1^{2} \mathrm{~T}_{2 \mathrm{~g}}$ state than for $1^{2} \mathrm{~T}_{1 \mathrm{u}}$ state $\left(\mathrm{SO}\left(1^{2} \mathrm{~T}_{2 \mathrm{~g}}\right)=0.11 \mathrm{eV}\right.$ and $\left.\mathrm{SO}\left(1^{2} \mathrm{~T}_{1 \mathrm{u}}\right)=0.04 \mathrm{eV}\right)$. In contrary to the $\operatorname{ADC}(3)$ method, the size of the SO splitting using the FSCC method for $1^{2} \mathrm{~T}_{1 \mathrm{u}}$ state is larger than for $1^{2} \mathrm{~T}_{2 \mathrm{~g}}\left(\mathrm{SO}\left(1^{2} \mathrm{~T}_{2 \mathrm{~g}}\right)=0.2 \mathrm{eV}\right.$ and $\left.\mathrm{SO}\left(1^{2} \mathrm{~T}_{1 \mathrm{u}}\right)=0.35 \mathrm{eV}\right)$. It seems that the FSCC method overestimates ionization potentials. In other words, due to the metal character of the $1^{2} \mathrm{~T}_{2 \mathrm{~g}}$ one would expect a larger SO splitting in comparison with $1^{2} \mathrm{~T}_{1 \mathrm{u}}$ where it has contributions of the $\mathrm{uO}_{\mathrm{p}}$, $\mathrm{uC}_{\mathrm{s}}, \mathrm{uC}_{\mathrm{p}}$ characters as obtained from the $\mathrm{ADC}(3)$ method.

In Table 3, we provide more results using the $\mathrm{ADC}(3)$ and FSCC methods at the relativistic and scalar relativistic levels of theory, which cover the outer valence IPs for the W(CO) 6 molecule. These results are computed in different levels of theory, where they are compared with the previous theoretical and experimental values. In general, the agreement between the computed results using the $A D C(3)-D C$ method and the previously reported experimental values are good. In this table, we also compared our results obtained from the $A D C(3)-D C$ with other reported computed IPs using the SAC-Cl and OVGF methods, where in the later the relativistic contribution was not taken into account in the calculation.

We present the simulated spectrum within the energy range of 5 and $27 \mathrm{eV}$ using the ADC(3) method employing the DC and DK Hamiltonians in Figure 2 and Figure 3, respectively, where the stick diagrams are convoluted by Lorentzian envelopes.

n Figure 2 and Figure 3, the pole strengths refer to the relative intensities in each line of the spectrum and the Mulliken population analysis reflecting contributions of molecular orbitals in the ionic states is also shown. The computed spectrum in this work represents five bands and position of each band is in a good agreement with the one reported in Ref. [4], measured in the energy range 5 to $25 \mathrm{eV}$. Especially, the broadening of the band in the energy range of 12 to $17 \mathrm{eV}$ is quite similar to the experimental spectrum measured in Ref. [4] and Figure 4. Furthermore the position and intensities of the last two bands are close to the experimental bands reported in Ref. [4]. The last band of the photoelectron spectrum in the energy range of 20 to $25 \mathrm{eV}$ is nicely reproduced and in consistent with the experimental result of Ref. [4]. The characterization of the first three bands can be explained as follows. The first, second, third, fourth and fifth bands of the photoelectron spectrum are located in the three following energy ranges; less than $10 \mathrm{eV}, 12-17$ $\mathrm{eV}, 17-18 \mathrm{eV}, 18-22 \mathrm{eV}$ and $22-25 \mathrm{eV}$, respectively. From Figure 2, it can be seen that the first region is ionization from the metal $d$ orbital, while the second and third regions are the ionizations from the $\mathrm{CO}$ outer and iner-valence orbitals. The fourth and fifth regions are the ionization from orbitals with dominant characters of $\mathrm{UO}_{\mathrm{p}}, \mathrm{O}_{\mathrm{s}}$ and $\mathrm{O}_{\mathrm{p}}$. Comparing Figure 2 and Figure 3 indicates that the contribution of SO coupling is more pronounced in the energy range less than $\approx 10 \mathrm{eV}$, where the first computed IP using DK Hamiltonian is split into the two IPs using the DC Hamiltonian as can be seen in Figure 1. 


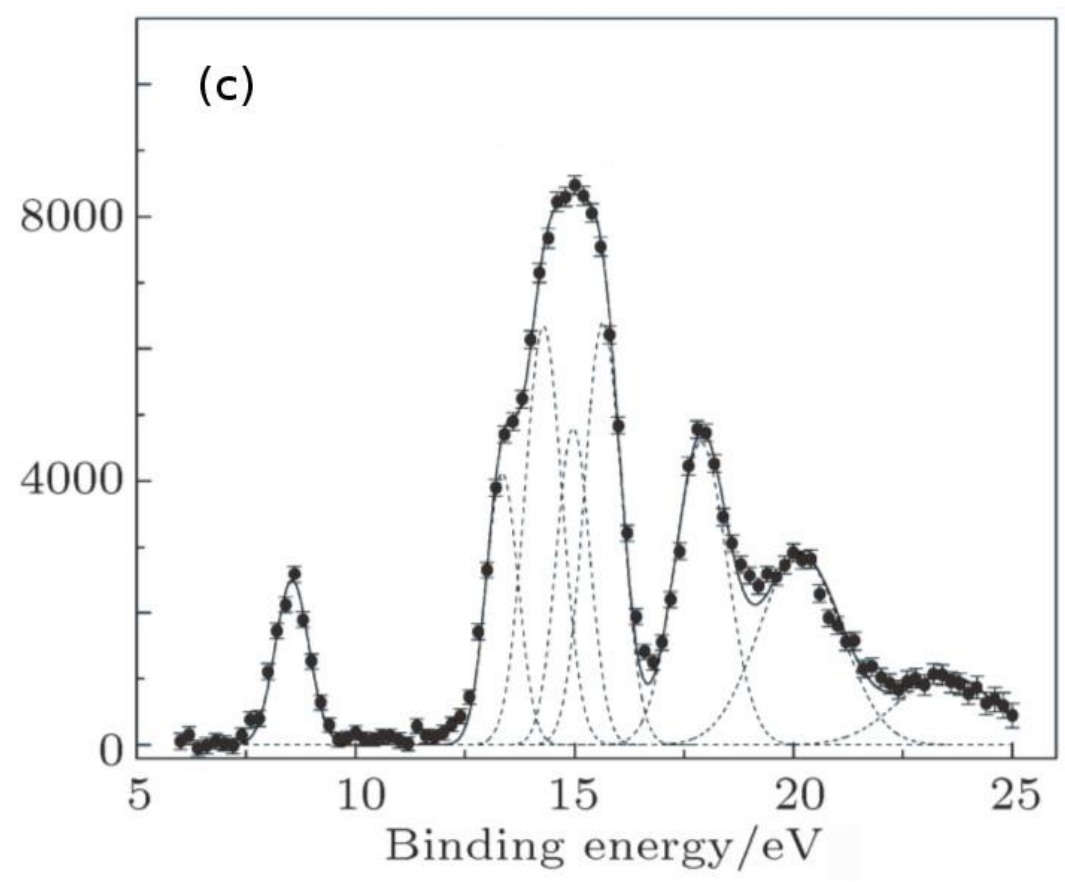

Figure 4 The experimental photoelectron spectrum of $\mathrm{W}(\mathrm{CO})_{6}$ which has been reprinted with permission from Lei et al, Chin. Phys. B. Copyright 2011 IOP science.

We also computed the double ionization spectrum of $\mathrm{W}(\mathrm{CO})_{6}$ using the $\mathrm{ADC}(2)$ method, where the corresponding results using the DK and DC Hamiltonians are shown in Figure 5a and Figure 5b, respectively. In this case, the pole strengths represent the intensities and the Mulliken population in this figure determines the contribution of molecular orbitals in the ionic states. Our calculation shows that the effect of the SO coupling plays an important role for better understanding of the double ionization spectrum. From the result shown in Figure $5 a$ and Figure $5 b$, it can be understood that there is a dominant contribution of $\mathrm{gW}_{\mathrm{d}}$ in both $\mathrm{DK}$ and $\mathrm{DC}$ cases and we can see an increase in the number of lines in the spectrum when the SO coupling is taken into account. The experimental value for the first double ionization energy is $22.90 \mathrm{eV}$ [26], which is close to our computed value $22.29 \mathrm{eV}$.
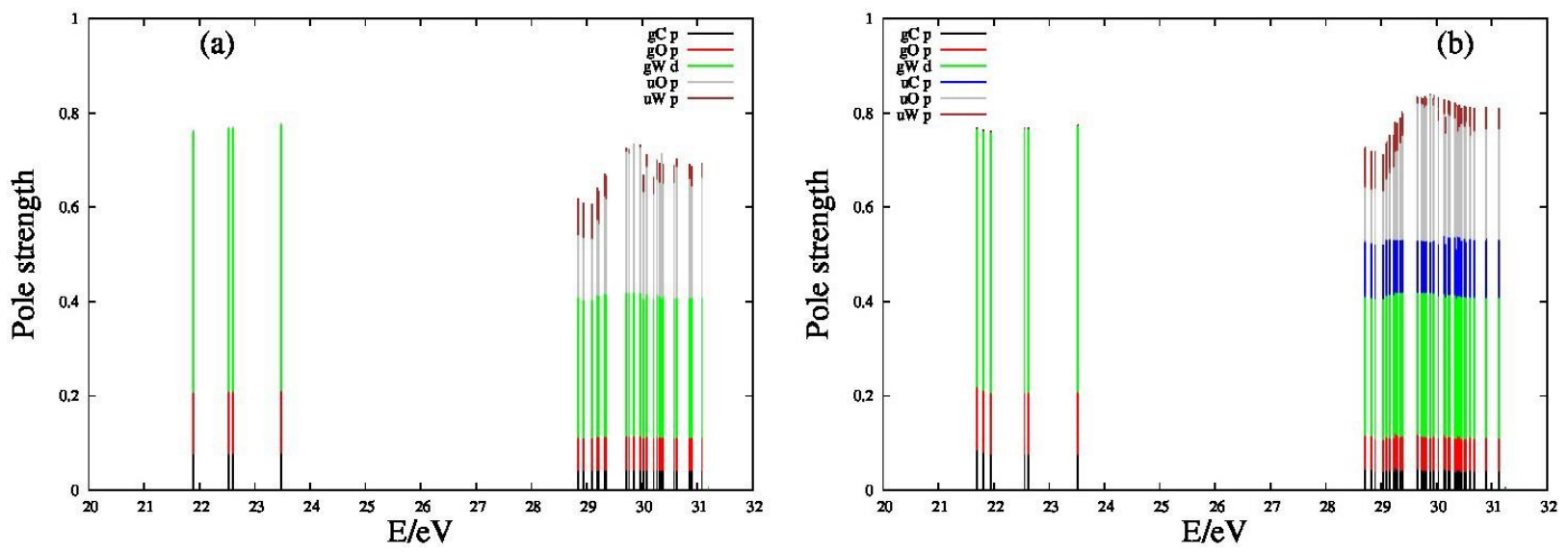

Figure 5 The computed double ionization potentials computed using $A D C(2)$ using $D K$ (a) and DC (b) Hamiltonians. 
Table 4 Vibrational Frequencies for $\mathrm{Cr}(\mathrm{CO})_{6}$ computed using ADF for the NR and SOZORA Hamiltonian, which are compared with the corresponding experimental values. The unit is $\mathrm{cm}^{-1}$

\begin{tabular}{llcl}
\hline NR & SO-ZORA & Assignment & Expt. $^{\mathrm{a}}$ \\
\hline 44 & 45.2 & $\mathrm{~T}_{2 \mathrm{u}}$ & - \\
78.75 & 80.14 & $\mathrm{~T}_{2 \mathrm{~g}}$ & - \\
96.82 & 97.96 & $\mathrm{~T}_{1 \mathrm{u}}$ & - \\
372.54 & 375.86 & $\mathrm{~T}_{1 \mathrm{~g}}$ & - \\
395.03 & 398.11 & $\mathrm{~A}_{1 \mathrm{~g}}$ & 381 \\
399.85 & 402.41 & $\mathrm{E}_{\mathrm{g}}$ & 394 \\
452.32 & 456.87 & $\mathrm{~T}_{1 \mathrm{u}}$ & - \\
523.78 & 526.7 & $\mathrm{~T}_{2 \mathrm{u}}$ & - \\
538.93 & 542.74 & $\mathrm{~T}_{2 \mathrm{~g}}$ & - \\
693.03 & 696.08 & $\mathrm{~T}_{1 \mathrm{u}}$ & - \\
1973.53 & 1972.22 & $\mathrm{~T}_{1 \mathrm{u}}$ & - \\
1994.56 & 1993.14 & $\mathrm{E}_{\mathrm{g}}$ & 2018 \\
2086.09 & 2085.33 & $\mathrm{~A}_{1 \mathrm{~g}}$ & 2112 \\
\hline
\end{tabular}

${ }^{\mathrm{a}}$ Ref. [27]

\section{$3.2 \mathrm{Cr}(\mathrm{CO})_{6}$}

The ground state equilibrium structure of $\mathrm{Cr}(\mathrm{CO})_{6}$ is computed by using different levels of theory as summarized in Table 1, where the computed bond lengths are compared with the available experimental results. It can be seen that the bond lengths computed at the SO-ZORA and 4c-DFT levels of theory are closer to the corresponding experimental results comparing with the other computational methods mentioned in Table 1. In Table 4, we also report the vibrational frequencies for $\mathrm{Cr}(\mathrm{CO})_{6}$ computed at the SO-SORA and NR levels of theory. As carried out for $\mathrm{W}(\mathrm{CO})_{6}$, the same computational methods are used for studying the electronic structure of this molecule (i.e., $\operatorname{ADC}(3)$ and FSCC methods using DK and DC Hamiltonians). The corresponding results are shown in Figure 6, Figure 7 and Figure 8 as well as Table 5, which are described in more details as follows. In Figure 6 for a DC-SCF calculation, one can observe that the $t_{2 g}$ orbital, composed of $\mathrm{gCr}_{\mathrm{d}}$ orbital, is split into the $\mathrm{e}_{\mathrm{g} 5 / 2}$ and $\mathrm{u}_{\mathrm{g} 3 / 2}$ spinors by amount of $0.15 \mathrm{eV}$, which is reduced by more than factor of half in comparison with the splitting of the $1^{2} \mathrm{~T}_{2 \mathrm{~g}}$ state in $\mathrm{W}(\mathrm{CO})_{6}$. The Mulliken population analysis reveals that the $t_{2 g}$ orbital has a considerable $\mathrm{gCr}_{\mathrm{d}}$ contribution of $70 \%$ in the DK cases, which is responsible for the splitting of the $t_{2 g}$ orbitals compared to the $\mathrm{gCr}_{\mathrm{d}}$ contribution of $50 \%$ and $71 \%$ in the $\mathrm{e}_{\mathrm{g} 5 / 2}$ and $\mathrm{u}_{\mathrm{g} 3 / 2}$ orbitals, respectively. This considerable 
$\mathrm{gCr}_{\mathrm{d}}$ population of the ground state with $\mathrm{t}_{2 \mathrm{~g}}$ symmetry leads to the SO splitting of 0.10 and 0.05 $\mathrm{eV}$ with respect to the DK value. In comparison with $\mathrm{W}(\mathrm{CO})_{6}$, the size of SO splitting is reduced by factor of half.

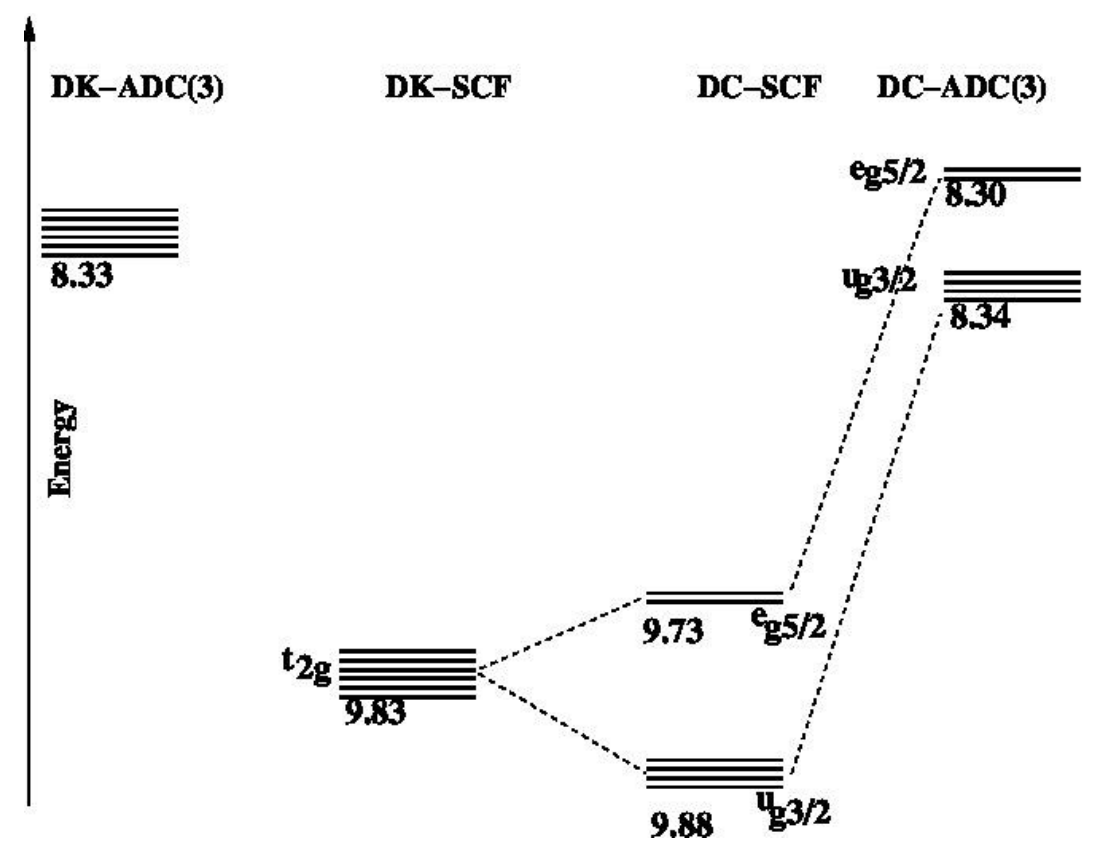

Figure 6 Energy level diagram for $\operatorname{Cr}(\mathrm{CO})_{6}$ calculated at the uncorrelated (SCF) and correlated $(A D C(3))$ level of theory for the DC and DK Hamiltonians in eV. The energy values correspond to the IPs.

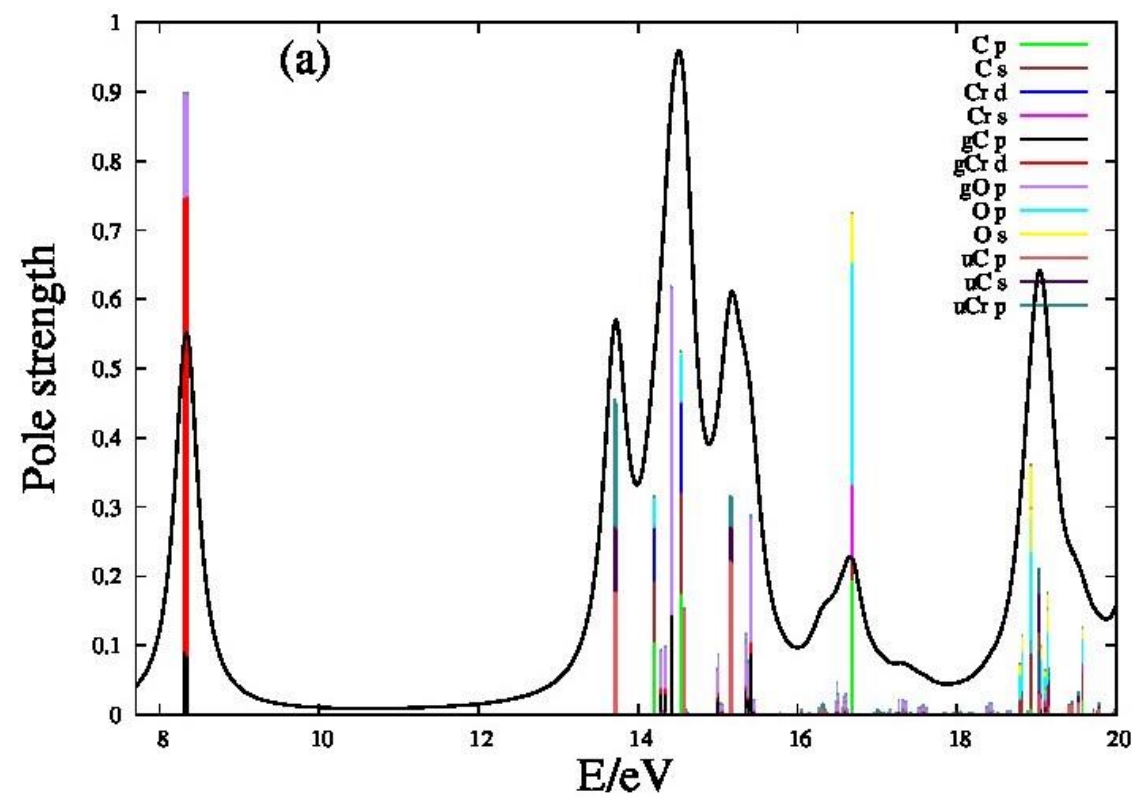

Figure 7 The simulated photoelectron spectrum of $\mathrm{Cr}(\mathrm{CO})_{6}$ calculated by employing the DC-ADC(3) method. 


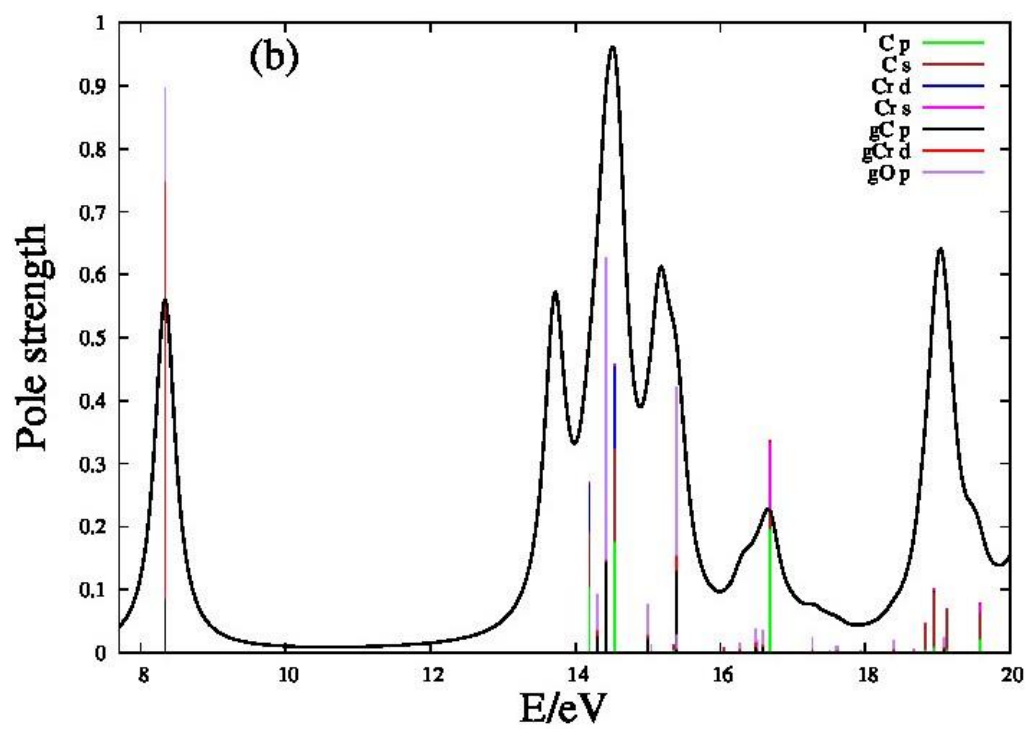

Figure 8 The simulated photoelectron spectrum of $\mathrm{Cr}(\mathrm{CO})_{6}$ calculated by employing DK-ADC(3) method.

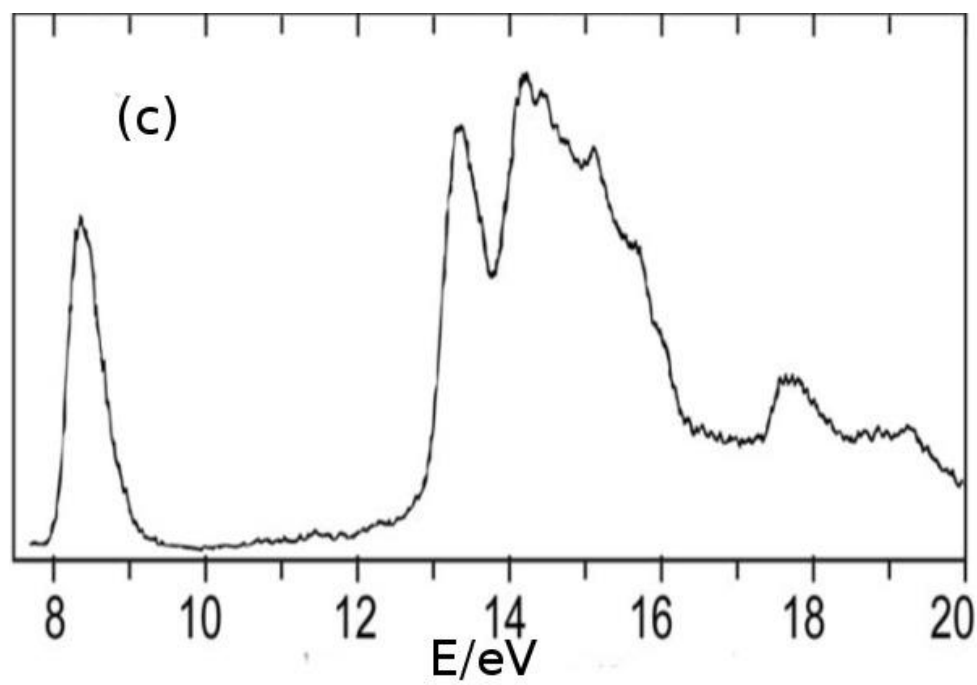

Figure 9 The experimental spectrum which has been reprinted with permission from B.R. Higginson et al, Faraday Trans. 2 69, 1659 1973. Copyright 1973 American Chemical Society.

Table 5 Ionization potentials of $\mathrm{Cr}(\mathrm{CO})_{6}$ for the valence spinors at the scalar and fully relativistic levels of theory which correspond to the DK and DC Hamiltonians, respectively. Note that these results are calculated by using the $A D C(3)$ and FSCC methods. All values are in $\mathrm{eV}$. The values in the parentheses refer to the differences between the computed IPs with the corresponding experimental values reported in Ref. [23]

\begin{tabular}{clllllll}
\hline \multirow{2}{*}{ state } & $\begin{array}{l}\text { ADC(3)- } \\
\text { DK }\end{array}$ & $\begin{array}{l}\text { FSCC- } \\
\text { DK }\end{array}$ & ADC(3)-DC & $\begin{array}{l}\text { FSCC- } \\
\text { DC }\end{array}$ & $\begin{array}{l}\text { SAC-Cl(with } \\
\text { SO) }\end{array}$ & ADC(3) & \multirow{2}{*}{ expt. $^{\mathbf{c}}$} \\
\hline $1^{2} \mathrm{~T}_{2 \mathrm{~g}}$ & 8.33 & 8.39 & $8.30(0.1)$ & 8.37 & $8.55(-0.15)$ & 9.15 & 8.4 \\
& & & 8.34 & 8.4 & 8.59 & &
\end{tabular}




\begin{tabular}{|c|c|c|c|c|c|c|c|}
\hline \multirow[t]{2}{*}{$1^{2} \mathrm{~T}_{1 \mathrm{u}}$} & 13.71 & 14.15 & $\begin{array}{l}13.70(- \\
0.32)\end{array}$ & 14.14 & $13.76(-0.38)$ & \multirow[t]{2}{*}{14.06} & \multirow[t]{2}{*}{13.38} \\
\hline & & & 13.73 & 14.17 & 13.78 & & \\
\hline \multirow[t]{2}{*}{$1^{2} \mathrm{~T}_{1 \mathrm{~g}}$} & 14.19 & - & $\begin{array}{l}14.19 \\
(0.02)\end{array}$ & - & 14.58 & \multirow[t]{2}{*}{$\begin{array}{l}14.75(- \\
0.37)\end{array}$} & \multirow[t]{2}{*}{ 14. 21} \\
\hline & & & 14.28 & - & 14.6 & & \\
\hline $1^{2} E_{g}$ & 14.3 & - & 14.35 & - & 14.81 & $14.79,15.14$ & \multirow{3}{*}{14.4} \\
\hline \multirow[t]{2}{*}{$1^{2} \mathrm{~T}_{2 \mathrm{u}}$} & 14.41 & - & $\begin{array}{l}\text { 14.41(- } \\
0.01)\end{array}$ & - & $14.83(-0.43)$ & 14.94 & \\
\hline & & & 14.42 & - & 14.85 & & \\
\hline \multirow[t]{2}{*}{$1^{2} T_{2 g}$} & 14.53 & - & $14.53(0.59)$ & - & $15.47(-0.59)$ & 15.71 & \multirow[t]{2}{*}{15.12} \\
\hline & & & 14.57 & - & 15.48 & & \\
\hline \multirow[t]{2}{*}{$1^{2} \mathrm{~T}_{1 \mathrm{u}}$} & 14.58 & - & 14.58 & - & 15.48 & 15.51 & \\
\hline & & & 14.71 & - & 15.51 & & \\
\hline $1^{2} A_{1 g}$ & 15.1 & - & $15.10(0.50)$ & - & 16.19 & $\begin{array}{l}16.77(- \\
1.17)\end{array}$ & 15.6 \\
\hline
\end{tabular}

${ }^{\text {aRef. [24] }}$

${ }^{\mathrm{b}}$ Ref. [28]

'Ref. [23]

In the next step, we treat the electron correlation utilizing the $\mathrm{ADC}(3)$ and FSCC methods using the DC and DK Hamiltonians. In Figure 6, the $A D C(3)$ calculation using the DK Hamiltonian results in the first IP which is related to an ionization from the triply degenerate orbital with the $t_{2 g}$ symmetry. In the four-component framework, due to the so coupling, the triply degenerate orbital undergoes a splitting leading to two different IPs, which are assigned to $\mathrm{e}_{\mathrm{g} 5 / 2}$ and $\mathrm{u}_{\mathrm{g} 3 / 2}$. It can be seen that both $A D C(3)$ and FSCC methods yields nearly the similar sizes of SO splitting for $1^{2} \mathrm{~T}_{2 \mathrm{~g}}$ and $1^{2} \mathrm{~T}_{1 \mathrm{u}}$ states $(\approx 0.03 \mathrm{eV})$, see from Table 5 and Figure 7. The $1^{2} \mathrm{~T}_{2 \mathrm{~g}}$ and $1^{2} \mathrm{~T}_{1 \mathrm{u}}$ has considerable $\mathrm{gCr}_{\mathrm{d}}$ and $\mathrm{uCr}_{\mathrm{p}}$ contributions, respectively, which can explain the so splitting for these two states. In contrary to $\mathrm{W}(\mathrm{CO})_{6}$ where the largest SO splitting exists for $1^{2} \mathrm{~T}_{2 \mathrm{~g}}$ state, the results in Table 5 show that the largest SO splitting for $\mathrm{Cr}(\mathrm{CO})_{6}$ is for the $1^{2} \mathrm{~T}_{1 \mathrm{~g}}$ state $(0.09 \mathrm{eV})$, which has $\mathrm{gCr}_{\mathrm{d}}, \mathrm{uO}_{\mathrm{p}}$ and $\mathrm{gO}_{\mathrm{p}}$ contributions. In Table 5, we also compare our results with the available experimental and theoretical results. The previous $A D C(3)$ calculation of Ref. [28] was carried out at the scalar relativistic level of theory, where the SO coupling was not treated. Furthermore in Ref. [24], the IPs were computed using the SAC-CI method considering the SO coupling. Our $A D C(3)$ results with considering SO coupling are close to the experimental ones, while we can see more deviations (especially for the lowest and highest IPs) from the experimental results using the SAC-Cl and $A D C(3)$ method from Refs. [24, 28].

In Figure 7 and Figure 8, we also present the ionization spectrum using the $A D C(3)$ method with employing the DC and DK Hamiltonians, respectively. The agreement between the computed spectrum in Figure 7 and the experimental one is good. In Figure 7, the splitting between the ionization peaks due to presence of the SO coupling can be clearly seen while we do not see such a feature in Figure 8, where we compute spectrum at the scalar relativistic level of theory. The effect of SO coupling is more pronounced in the low energy part of the ionization spectrum than in the middle and high energy parts of the spectrum. This observation is consistent with this fact that characterization of the IPs in the energy range of less than $14.5 \mathrm{eV}$ has a metal character, while for 
the energy range larger than $14.5 \mathrm{eV}$ contributions of molecular orbitals in the ionic states mostly depend on the orbitals of light elements $\mathrm{C}$ and $\mathrm{O}$ atoms. Thus, this can be considered as a reason why the size of SO splitting in the low and middle energy parts of spectrum is larger than the high energy parts of the spectrum.

We also computed the double ionization spectrum for this molecule using the ADC(2) method in Figure 10. We can see that the role of the SO coupling is important in the double ionization spectrum. This is due to the fact that the DIPs have mostly the metal character leading to the splitting of DIPs.
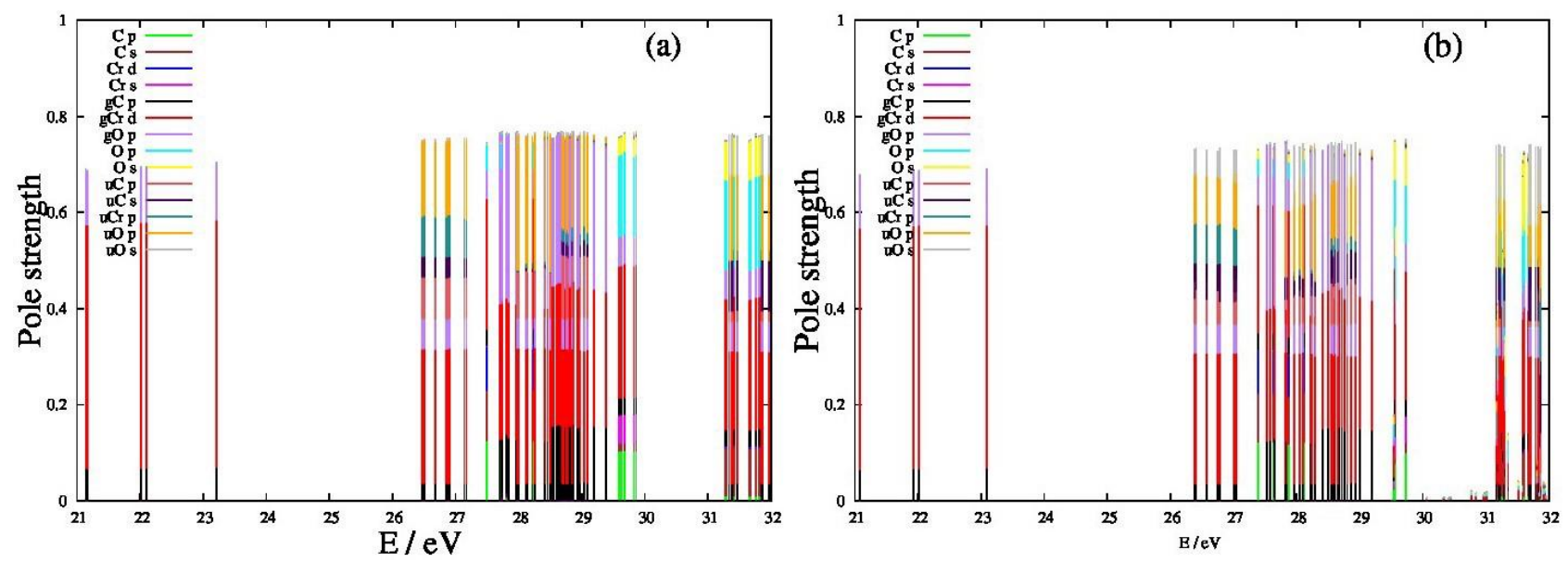

Figure 10 The computed double ionization potentials computed using $A D C(2)$ using DC (a) and DK (b) Hamiltonians.

\section{Conclusions}

In this study, we computed the SIP and DIP spectra for the two complexes $\mathrm{M}(\mathrm{CO})_{6}$ with $\mathrm{M}=$ $(\mathrm{W}, \mathrm{Cr}$ ) employing the four-component $\mathrm{ADC}(2), \mathrm{ADC}(3)$ and $\mathrm{FSCC}$ methods. For all complexes under consideration an acceptable agreement with the experimental single and double ionization spectra could be achieved. For the first time we computed the DIP spectra for both complexes at the scalar relativistic and four-component levels of theory. The computed photoelectron spectra are in good agreement with the corresponding experimental ones. For $\mathrm{W}(\mathrm{CO})_{6}$, due to the similarity between the computed spectrum and the measured one in Ref. [4], the conflicting situation available between the two reported experimental spectra in Refs. $[3,4]$ is thus resolved. It is found that the role of the SO coupling is important for a thorough understanding of the SIP and DIP spectra of both complexes.

\section{Acknowledgments}

This research was enabled by support provided by Westgrid(www.westgrid.ca) and Compute Canada(www.computecanada.ca). We also pleased to thank Dr. S. Zarrini for his critical suggestions. One of us (BN) is indebted to the Beilstein-Institut for the financial support.

\section{Author Contributions}


Behnam Nikoobakht: carrying out all computations, interpretations and analysis of the results, writing the manuscript. Gulzari L Malli and Martin Siegert: discussion on the results and helping in writing the manuscript.

\section{Competing Interests}

The authors have declared that no competing interests exist.

\section{References}

1. Bartlett RJ, Musiał M. Coupled-cluster theory in quantum chemistry. Rev Mod Phys. 2007; 79: 291.

2. Nilsson A, Mårtensson N, Svensson S, Karlsson L, Nordfors D, Gelius U, et al. High resolution xray photoelectron spectroscopy study of $\mathrm{Cr}(\mathrm{CO})_{6}$ in the gas phase. J Chem Phys. 1992; 96: 8770-8780.

3. Higginson BR, Lloyd DR, Evans S, Orchard AF. Photoelectron studies of metal carbonyls. Part 5. - Substituted group VIIA carbonyls. J Chem Soc Faraday Trans. 1975; 71: 1913-1928.

4. Le-Lei S, Kun L, Zhi-Hong L, Chuan-Gang N, Jing-Kang D. Valence orbitals of $W(C O)_{6}$ using electron momentum spectroscopy. Chin Phys B. 2011; 20: 113403.

5. Nikoobakht B. ${ }^{2} \mathrm{~T}_{2 \mathrm{~g}} \leftarrow{ }^{1} \mathrm{~A}_{1 \mathrm{~g}}$ photo-electron spectrum of octahedral tungsten hexacarbonyl. Phys Chem Chem Phys. 2016; 18: 33357-33368.

6. Ham FS. Dynamical Jahn-Teller effect in paramagnetic resonance spectra: Orbital reduction factors and partial quenching of spin-orbit interaction. Phys Rev. 1965; 138: A1727.

7. Dunning Jr TH. Gaussian basis sets for use in correlated molecular calculations. I. The atoms boron through neon and hydrogen. J Chem Phys. 1989; 90: 1007-1023.

8. DIRAC, a relativistic ab initio electronic structure program, Release DIRAC14 (2014), written by L. Visscher, H. J. Aa. Jensen, R. Bast, and T. Saue, with contributions from V. Bakken, K. G. Dyall, S. Dubillard, U. Ekstr"om, E. Eliav, T. Enevoldsen, E. Faßhauer, T. Fleig, O. Fossgaard, A. S. P. Gomes, T. Helgaker, J. K. Lærdahl, Y. S. Lee, J. Henriksson, M. Ilia`s, Ch. R. Jacob, S. Knecht, S. Komorovsky', O. Kullie, C. V. Larsen, H. S. Nataraj, P. Norman, G. Olejniczak, J. Olsen, Y. C. Park, J. K. Pedersen, M. Pernpointner, K. Ruud, P. Sal ek, B. Schimmelpfennig, J. Sikkema, A. J. Thorvaldsen, J. Thyssen, J. van Stralen, S. Villaume, Visser, T. Winther, and S. Yamamoto (see http://www.diracprogram.org).

9. Te Velde GT, Bickelhaupt FM, Baerends EJ, Fonseca Guerra C, van Gisbergen SJ, Snijders JG, et al. Chemistry with ADF. J Comp Chem. 2001; 22: 931-967.

10. Van Lenthe E, Snijders JG, Baerends EJ. The zero-order regular approximation for relativistic effects: The effect of spin-orbit coupling in closed shell molecules. J Chem Phys. 1996; 105: 6505-6516.

11. Van Lenthe E, Baerends EJ, Snijders JG. Relativistic total energy using regular approximations. J Chem Phys. 1994; 101: 9783-9792.

12. Van Lenthe E, Baerends EJ, Snijders JG. Relativistic regular two-component Hamiltonians. J Chem Phys. 1993; 99: 4597-4610.

13. Van Lenthe E, Baerends EJ. Optimized Slater-type basis sets for the elements 1-118. J Comp Chem. 2003; 24: 1142-1156. 
14. Iliaš M, Pershina V. Hexacarbonyls of Mo, W, and Sg: Metal-CO bonding revisited. Inorg Chem. 2017; 56: 1638-1645.

15. Jost A, Rees B, Yelon WB. Electronic structure of chromium hexacarbonyl at $78 \mathrm{KI}$ Neutron diffraction study. Acta Crystallographica Section B. 1975; 31: 2649-2658.

16. Pershina $\mathrm{V}$, Anton J. Theoretical predictions of properties and gas-phase chromatography behaviour of carbonyl complexes of group-6 elements $\mathrm{Cr}$, Mo, W, and element 106, Sg. J Chem Phys. 2013; 138: 174301.

17. Visscher L, Eliav E, Kaldor U. Formulation and implementation of the relativistic Fock-space coupled cluster method for molecules. J Chem Phys. 2002; 115: 9720-9726.

18. Pernpointner M. A fully relativistic study of the $\mathrm{Pt}(\mathrm{CN})_{4}{ }^{2}$-and $\mathrm{Pt}(\mathrm{CN})_{6}{ }^{2-}$ photodetachment spectra. Chem Phys. 2007; 338: 44-52.

19. Malli GL. Dirac-Fock-Breit-Gaunt calculations for tungsten hexacarbonyl W(CO) $)_{6}$ J Chem Phys. 2016; 144: 194301.

20. Dyall KG. An exact separation of the spin-free and spin-dependent terms of the DiracCoulomb-Breit Hamiltonian. J Chem Phys. 1994; 100: 2118-2127.

21. Dyall KG. Relativistic double-zeta, triple-zeta, and quadruple-zeta basis sets for the $4 \mathrm{~s}, 5 \mathrm{~s}, 6 \mathrm{~s}$, and 7s elements. J Phys Chem A. 2009; 113: 12638-12644.

22. Braterman PS. Metal carbonyl spectra. London: Academic Press; 1975.

23. Higginson BR, Lloyd DR, Burroughs P, Gibson DM, Orchard AF. Photoelectron studies of metal carbonyls. Part 2. - The valence region photoelectron spectra of the group VIA hexacarbonyls. J Chem Soc Faraday Trans 2. 1973; 69: 1659-1668.

24. Fukuda R, Hayaki S, Nakatsuji H. Valence ionization spectra of group six metal hexacarbonyls studied by the symmetry-adapted cluster-configuration interaction method. J Chem Phys. 2009; 131: 174303.

25. Bayse CA, Ortwine KN. Calculation of photoelectron spectra of molybdenum and tungsten complexes using Green's functions methods. J Phys Chem A. 2007; 111: 7841-7847.

26. Wnorowski K, Stano M, Barszczewska W, Jówko A, Matejčík Š. Electron ionization of W(CO) 6 : Appearance energies. Int J Mass Spectrom. 2012; 314: 42-48.

27. Khan FA, Clemmer DE, Schultz RH, Armentrout PB. Sequential bond energies of chromium carbonyls ( $\mathrm{Cr}(\mathrm{CO}) \mathrm{x}+, \mathrm{x}=1-6)$. J Phys Chem. 1993; 97: 7978-7987.

28. Ohno M, von Niessen W, Tarantelli F. Many-body calculation of the valence photoemission spectrum of $\mathrm{Cr}(\mathrm{CO})_{6}$. Phys Rev B. 1992; 45: 1851. 


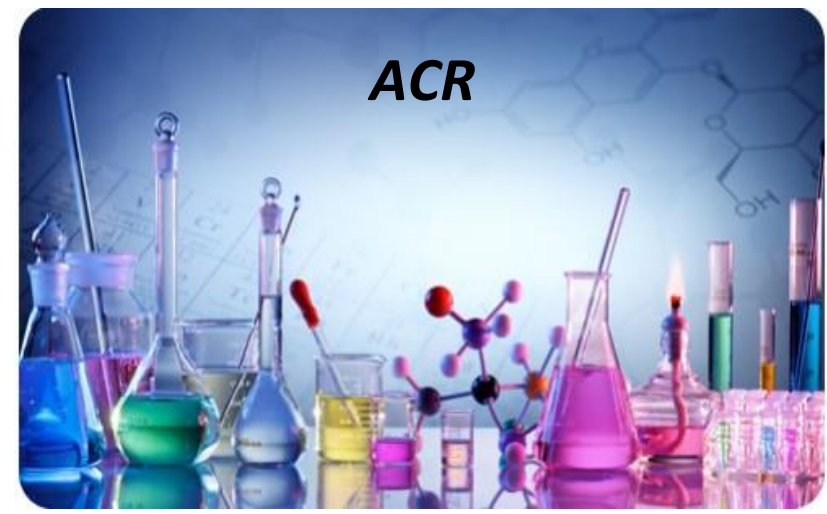

Enjoy $A C R$ by:

1. Submitting a manuscript

2. Joining in volunteer reviewer bank

3. Joining Editorial Board

4. Guest editing a special issue

For more details, please visit:

http://www.lidsen.com/journals/acr 